\title{
Gap effect and reaction time distribution: simple vs choice manual responses
}

W. Machado-Pinheiro ${ }^{1,3}$,

L.G. Gawryszewski² and L.E. Ribeiro-do-Valle ${ }^{3}$

\author{
1Departamento de Fisiologia, Instituto Biomédico, and \\ ${ }^{2}$ Departamento de Neurobiologia, Instituto de Biologia, \\ Universidade Federal Fluminense, Niterói, RJ, Brasil \\ ${ }^{3}$ Departamento de Fisiologia e Biofísica, Instituto de Ciências Biomédicas, \\ Universidade de São Paulo, São Paulo, SP, Brasil
}

\section{Correspondence \\ W. Machado-Pinheiro Departamento de Fisiologia Instituto Biomédico, UFF Rua Hernani Mello, 101 24210-130 Niterói, RJ Brasil \\ Fax: +55-21-620-5266 \\ E-mail: mflwmp@vm.uff.br}

Research supported by CN Pq, CAPES, FINEP, FAPERJ, PROPP-UFF and PRONEX/MCT.

Publication supported by FAPESP.

$W$. Machado-Pinheiro is the recipient of a CAPES fellowship. ......................

Received January 20, 1998 Accepted July 27, 1998

\section{Abstract}

It is well known that saccadic reaction times (SRT) are reduced when the target is preceded by the offset of the fixation point (FP) - the gap effect. Some authors have proposed that the FP offset also allows the saccadic system to generate a separate population of SRT, the express saccades. Nevertheless, there is no agreement as to whether the gap effect and express responses are also present for manual reaction times (MRT). We tested the gap effect and the MRT distribution in two different conditions, i.e., simple and choice MRT. In the choice MRT condition, subjects need to identify the side of the stimulus and to select the appropriate response, while in the simple MRT these stages are not necessary. We report that the gap effect was present in both conditions (22 ms for choice MRT condition; $15 \mathrm{~ms}$ for simple MRT condition), but, when analyzing the MRT distributions, we did not find any clear evidence for express manual responses. The main difference in MRT distribution between simple and choice conditions was a shift towards shorter values for simple MRT.
It is well known that when a motor response to a visual stimulus is preceded by a warning signal its latency is reduced (1). Moreover, when the warning signal is the offset of a light stimulus, some special phenomena appear. Saslow (2) showed that if a fixation point (FP) is turned off some time before the onset of an imperative stimulus (the gap paradigm) the saccadic reaction time (SRT) decreases in relation to the condition with no FP offset (overlap paradigm) or with simultaneous FP offset and stimulus onset (gap 0). This reduction of SRT in the gap paradigm was called the "gap effect" by
Key words

- Attention

- Reaction time

- Gap effect

- Vision

- Express responses
Fischer and co-workers (for details, see Ref. 3).

Many investigators have confirmed the presence of the gap effect for SRT (3-12). Some have proposed that the gap paradigm reduces saccade latency and also facilitates the appearance of a very short latency population in the SRT distribution, the so-called express saccades (ES) (for a review, see Ref. 3). However, the universality of ES is still controversial. Several investigators, though observing a reduction of SRT in the gap paradigm, could not find a population of short latency responses. They suggested that 
Figure 1 - A, Schematic representation of stimulus display; $\mathrm{B}$, temporal sequence of occurrence of the fixation point (FP), the first stimulus or attention point (AP), and the imperative stimulus (S2)

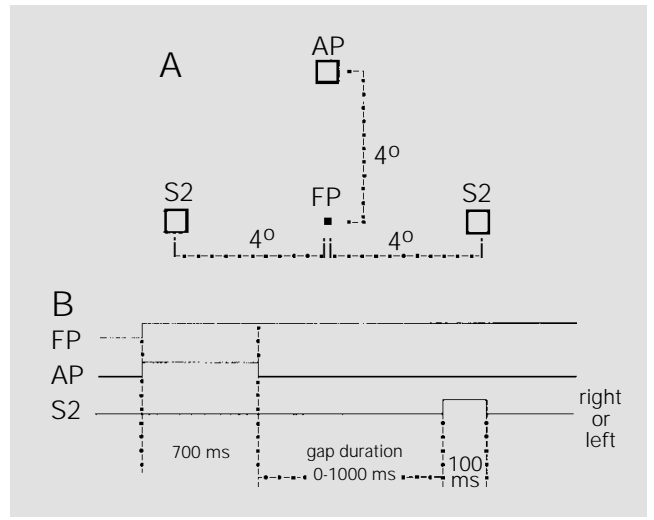

it would be more fruitful to focus attention on the gap effect per se, whether or not ES are present in the SRT distribution $(9,11$ 14). Thus, there are grounds to suggest that the gap effect and ES are distinct phenomena.

A very interesting question is whether the gap effect exists for manual reaction times (MRT). Reuter-Lorenz et al. (11) did not find the effect; Kingstone and Klein (9) found some latency facilitation, but attributed it to factors other than the gap effect. Fischer and Rogal (15), Ross and Ross (16), Iwasaki (8) and Bekkering et al. (4) found a gap effect for choice manual responses which, however, was proportionally smaller than that for SRT.

Express manual responses have not yet been demonstrated. Many investigators $(8,11$, $15,17,18)$ tried to find such a population of manual responses using the gap paradigm. In their studies, a choice MRT was used to avoid an anticipatory behavior during the experiments but no express manual responses were observed.

The aim of the present study was to determine how the offset of a peripheral visual stimulus to which the subject is paying attention modifies the MRT to a second visual stimulus. We employed a non-blocked array of gap durations to investigate the gap effect and the MRT distributions in two conditions, i.e., simple and choice MRT.

Eight volunteers (four males and four females) participated in this study. All of them were right-handed according to the Edinburgh Inventory (19), had normal or corrected vision, and their ages ranged from 20 to 24 years. The subjects were tested in a sound-attenuated room under dim ambient light. They sat in front of a CRT screen driven by a PC-486 microcomputer, which timed the stimuli and recorded the MRT. The head was positioned on a head-and-chin rest so that the distance between the eyes and the screen was approximately $57 \mathrm{~cm}$. Before data collection, subjects were submitted to some training, where the importance of maintaining fixation was stressed. During this training session, eye movements were monitored by an experimenter sitting behind the subject using a suitably oriented mirror.

Each trial began with the simultaneous presentation of a central point (fixation point - FP) and a square (attention point - AP, $0.5^{\circ}$ x $0.5^{\circ}$ ) located $4^{\circ}$ above it. After $700 \mathrm{~ms}$, AP went off and after a variable gap duration the imperative stimulus (an identical square S2) flashed for $100 \mathrm{~ms} 4^{\circ}$ to the left or to the right of the FP. There were three possible gap durations between the offset of AP and the onset of S2, i.e. 0,250 or $1000 \mathrm{~ms}$, which occurred randomly and with the same probability throughout the experiment (Figure 1). In addition, the gap duration of $1000 \mathrm{~ms}$ varied randomly from 800 to $1200 \mathrm{~ms}$. The subjects were instructed to fixate the FP and to pay attention to AP, because its offset was the cue that $\mathrm{S} 2$ would occur at any moment. There were two conditions: a) simple MRT, in which subjects had to press a key with the right index finger as soon as they detected the imperative stimulus (S2), irrespective of whether it occurred to the left or to the right of the FP. b) Choice MRT, in which subjects had to press the key spatially corresponding to the side of the stimulus (left or right) with the corresponding index finger (left or right, respectively), as soon as they detected $\mathrm{S} 2$. After each manual response, the latency (in milliseconds) appeared on the screen for $1000 \mathrm{~ms}$. Stimulus luminance was $11.5 \mathrm{~cd} /$ 
$\mathrm{m}^{2}$, and background luminance was $0.2 \mathrm{~cd} /$ $\mathrm{m}^{2}$. All subjects performed four sessions of 300 trials (four blocks of 75 trials with some minutes of rest between them) on separate days. Fifty MRT for each gap duration and side were recorded daily. The first session was only for training and its data were not considered. On the second and third days, the subjects performed the choice MRT condition, and on the fourth day, the simple MRT condition. MRT shorter than $100 \mathrm{~ms}$ or longer than $700 \mathrm{~ms}$ were considered to be errors (anticipations and slow responses, respectively) and were discarded. In the choice MRT condition, pressing the key not spatially corresponding to $\mathrm{S} 2$ was also considered an error. When one of these errors occurred, instead of the MRT, the message "anticipation" or "slow response" or "error" appeared on the screen for $1000 \mathrm{~ms}$. All error-trials were repeated at the end of each session. They corresponded to $2.98 \%$ in all trials.

The medians of the MRT obtained on the second, third and fourth days were submitted to an analysis of variance (ANOVA) in which day (second, third or fourth), visual hemifield (left or right) and gap duration (0, 250 or $1000 \mathrm{~ms}$ ) were used as within-subjects factors. The data were also submitted to post hoc analysis using the Newman-Keuls method. The level of significance adopted was $\mathrm{P}<0.05$.

ANOVA showed that day and gap duration were significant sources of variance $(\mathrm{F}(2,14)=27.378, \mathrm{P}<0.001$ and $\mathrm{F}(2,14)=$ 22.864, $\mathrm{P}<0.001$, respectively). Post hoc analysis revealed that the MRT observed on the second day (228 ms) did not differ significantly from that observed on the third day (222 ms), but these MRTs, which were obtained in the choice condition, were different from that observed on the fourth day, when simple MRT was used (204 ms). For the gap duration factor, the MRT for gap 0 (231 ms) was significantly different from those for gaps 250 and 1000 (211 and 212 ms, respectively), while these two did not differ significantly.

Since the MRTs for the second and third days were alike, we pooled the data in Figure 2A. This figure shows the variation of MRTs as a function of condition and gap duration. Note that the MRTs for gap 250 and for gap 1000 did not differ (217 and $219 \mathrm{~ms}$ for choice MRT; 200 and $198 \mathrm{~ms}$ for simple MRT, respectively), but both differed from the MRT for gap 0 under both conditions (240 and $215 \mathrm{~ms}$ for the choice and simple conditions, respectively). The differences between choice and simple MRT at each gap duration were almost the same, approximately $22 \mathrm{~ms}$. In short, it can be seen that a) for all gap durations choice MRTs were longer than simple MRTs, and b) MRTs for gap 0 were longer than those for gaps 250 and 1000 under both conditions.

Interaction was also observed between day and hemifield $(\mathrm{F}(2,14)=5.012, \mathrm{P}=$ 0.022 ). On the second and third days, when the choice condition was used, the latency of the responses to stimuli on the left visual field (225 and $219 \mathrm{~ms}$ for the second and third days, respectively) was shorter than the responses to stimuli in the right visual field (232 and $226 \mathrm{~ms}$, respectively). However, on the fourth day, when the simple condition was used, there was no significant difference between hemifields ( 205 and $203 \mathrm{~ms}$ for left and right targets, respectively).

We carried out a second ANOVA to determine whether the gap effect (difference between MRT of gap 0 and gap 250) differed between the choice and simple MRT conditions. In this ANOVA, gap effect was used as the dependent variable and condition (choice or simple reaction time) and visual hemifield (left or right) were used as withinsubject factors. ANOVA showed that the gap effect for the choice and the simple MRT condition was significantly different $(F(1,7)=5.877, P=0.044)$. The gap effect was stronger for the choice than for the simple MRT condition (22 vs $15 \mathrm{~ms}$ ), but 
Figure 2 - Main results of the experiment: $A$, Mean latency of manual reaction times (MRT) as a function of gap duration and condition (simple or choice) for the 8 subjects studied. The error bars indicate \pm 1 SEM. B, Distribution of MRTs for two subjects in the simple (upper graph) and choice (lower graph) M RT conditions. The number of manual responses (ordinate) is plotted as a function of reaction times (abscissa). A binwidth of $10 \mathrm{~ms}$ was used; the data from the right and left hemifields for each condition were pooled (100 manual responses for each curve). Solid lines, gap duration of $0 \mathrm{~ms}$; dotted lines, gap duration of 250 $\mathrm{ms}$; dashed lines, gap duration of $1000 \mathrm{~ms}$.

\section{A}

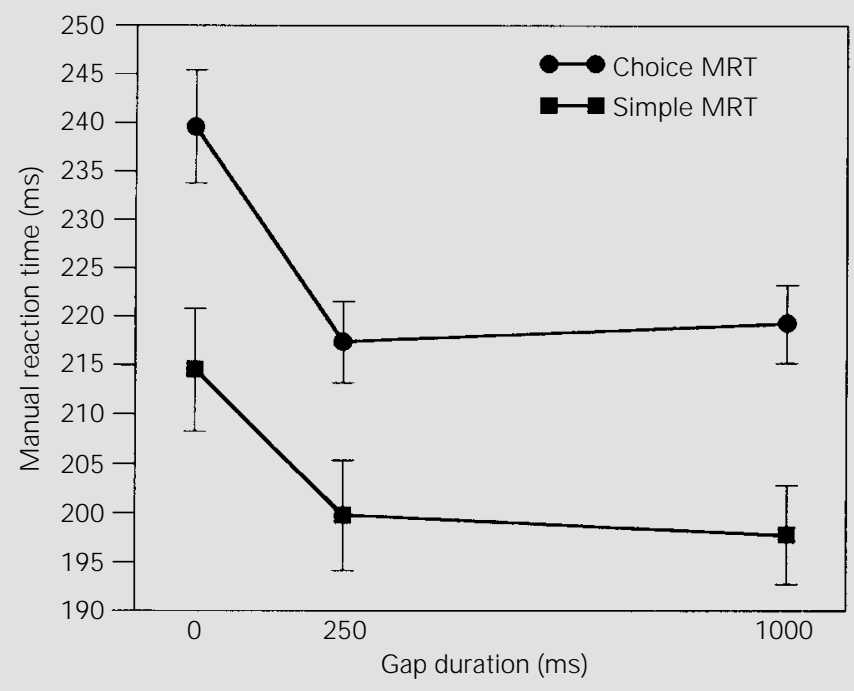

B

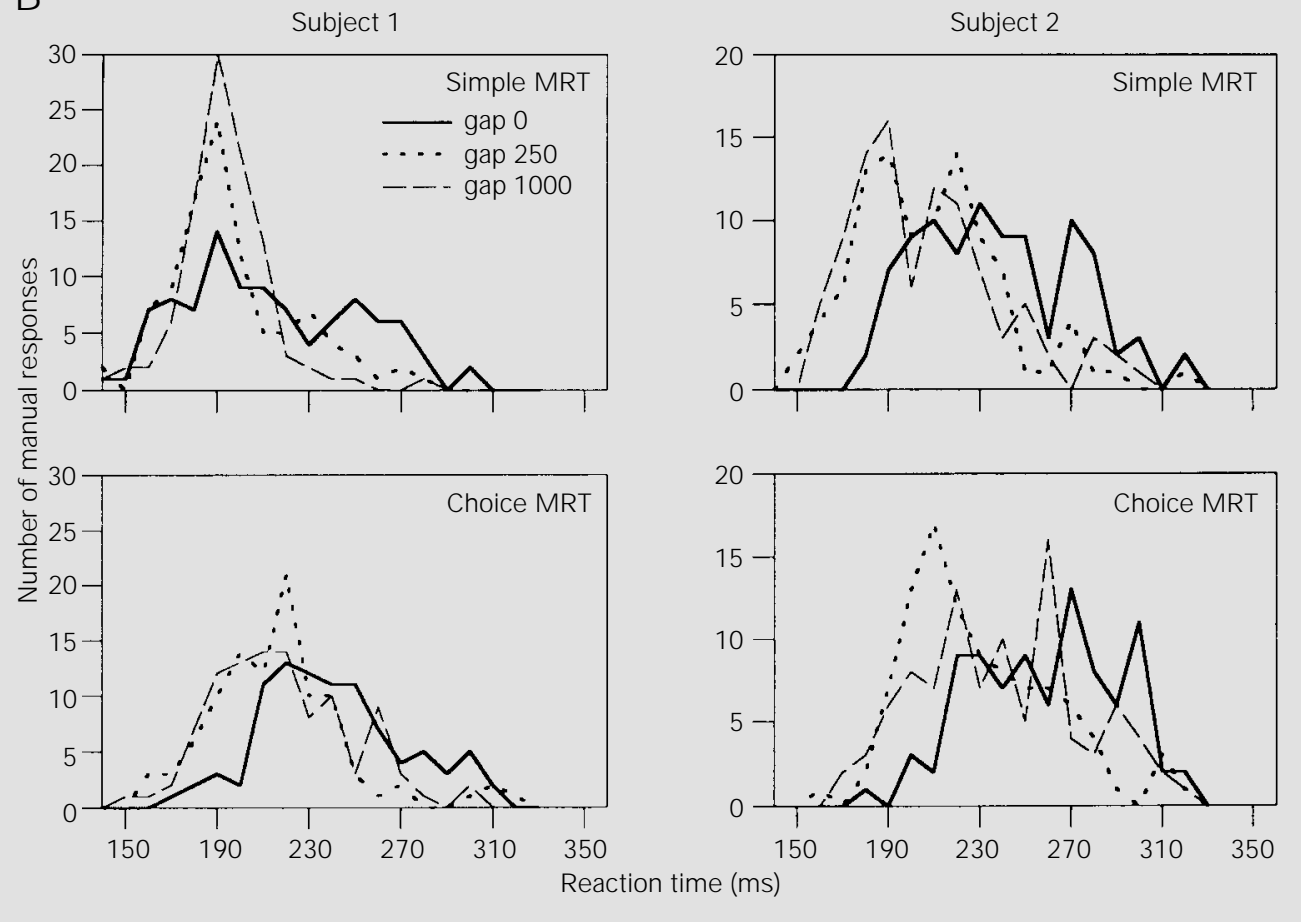

was present in both conditions.

MRT distributions were calculated for each of the eight subjects for all gap durations in both conditions. A binwidth of 10 ms was used. The first bin included MRTs from 140 to $149 \mathrm{~ms}$ and the last from 350 to 359. Figure 2B shows the MRT distributions for two subjects in the simple (upper panel) and choice MRT condition (lower panel) for gap durations of 0,250 and $1000 \mathrm{~ms}$. The plots of the simple condition were obtained using the data of the fourth day, and those of the choice condition using the data of the third day. Data from the left and right 
hemifields were pooled (100 MRTs for each curve). The tendency towards smaller values in the simple MRT condition in relation to the choice MRT condition observed in Figure 2A was also present in the MRT distributions of Figure 2B for all gap durations. Moreover, there was also a tendency towards smaller values for gaps 250 and 1000 compared to gap 0 for both conditions. There was no clear evidence for a separate subpopulation of MRT, equivalent to the express saccades. Only for two of the eight subjects studied can bimodality be suggested: for subject \#1 in the simple condition and gap 0 (solid line in the upper-left graph), and for subject \#2 in the simple condition and gaps 250 and 1000 (dotted and dashed lines in the upper-right graph). Subject \#1 showed a possible first population of MRTs around bin 190 and a second around bin 250 . The second and smaller peak could be seen only at a gap duration of $0 \mathrm{~ms}$, while the first, although existing for the three tested gap durations, was considerably higher at gap durations of 250 and $1000 \mathrm{~ms}$. Subject \#2 showed the two possible populations of MRT around bins 190 and 210-220, respectively, at gap durations of 250 and $1000 \mathrm{~ms}$. The other six subjects (data not shown) showed no sign of bimodality under any condition or gap duration tested. Therefore, bimodal distribution of response latencies, although suggested for some subjects, was not consistent in our sample. An intriguing fact is that, although the gap effect was stronger for the choice than for the simple MRT condition, the few bimodal MRT distributions we found were always in the simple condition. Therefore, we found a gap effect without a clear population of express manual responses. These results strongly support the idea that the gap effect and the express responses are distinct phenomena.

Fischer and Weber (for details, see Ref. 3) have considered the gap effect as the difference between reaction times in the overlap and the gap 200 condition. In the present study, we followed Saslow's (2) original approach and used the MRT at gap 0 (simultaneous AP offset and stimulus onset) as baseline for the gap effect. The gap effect was present in both the simple and choice MRT conditions and ranged from a mean value of $15 \mathrm{~ms}$ for the simple MRT to $22 \mathrm{~ms}$ for the choice MRT condition (see Figure $2 \mathrm{~A}$ ), suggesting a stronger gap effect for the choice MRT condition.

Bekkering et al. (4) found the gap effect for choice MRT but not for simple MRT and proposed that the gap effect is present only for spatially oriented responses. Our results show that the requirement to identify the stimulus location and to select the appropriate response increases MRT, i.e., choice MRTs are longer than simple MRTs. However, the response selection is not necessary to elicit the gap effect since this effect was found in both the choice and simple paradigms.

Our findings complement the data of others who used the choice MRT condition and a fixed array of gap durations and who were also unable to demonstrate express manual responses $(8,11,15,17,18)$. Therefore, over a wide range of conditions express manual responses could not be clearly demonstrated. If such a phenomenon does exist for manual responses the optimal conditions to generate manual express responses are yet to be found.

In short, we observed the gap effect for both simple and choice MRT without clear evidence of express MRT. In our view, the offset of AP facilitates the automatic orienting of attention to the target position at short gap durations. AP offset would elicit a disengagement of attention and, if the target occurs while the attention is disengaged (200$300 \mathrm{~ms}$ ), it is captured more quickly than if the attention is still engaged. This explanation, however, cannot be used for the gap duration of $1000 \mathrm{~ms}$ since the studies with SRT showed that the optimal interval for the gap effect is between 200 and $300 \mathrm{~ms}(5,10)$. 


\section{Acknowledgments}

We would like to thank Dr. Carlos Eduardo Rocha-Miranda (Academia Brasileira de Ciências) and Dr. Ronald Ranvaud (ICB-USP) for their valuable comments on an early ver- sion of the manuscript. We are also grateful to Mr. Antônio Romanazzi Vieira, from Programa de Geoquímica of the Universidade Federal Fluminense, who developed a detector of light stimuli for accurate determination of the time sequence in our experiments.

\section{References}

1. J ames W (1890/1950). The Principles of Psychology. Vol. 1. Authorized edition. Dover Publications Inc., New York.

2. Saslow MG (1967). Effects of components of displacement-step stimuli upon latency of saccadic eye movements. J ournal of the Optical Society of America, 57: 1024-1029.

3. Fischer B \& Weber H (1993). Express saccades and visual attention. Behavioral and Brain Sciences, 16: 553-610.

4. Bekkering $\mathrm{H}$, PrattJ \& Abrams RA (1996). The gap effect for eye and hand movement. Perception and Psychophysics, 58: 628-635.

5. Braun D \& Breitmeyer BG (1988). Relationship between directed visual attention and saccadic reaction times. Experimental Brain Research, 73: 546-552.

6. Fischer B \& Breitmeyer BG (1987). Mechanisms of visual attention revealed by saccadic eye movements. Neuropsychologia, 25: 73-83.

7. Fischer B \& Ramsperger E (1984). Human express saccades: extremely short reaction times of goal directed eye movements. Experimental Brain Research, 57: 191-195.
8. Iwasaki S (1990). Facilitation of reaction times with gap paradigm: comparison of manual and saccadic responses. Ergonomics, 33: 833-850.

9. Kingstone $A \& \&$ Klein RM (1993). Visual offsets facilitate saccadic latency: does predisengagement of visuospatial attention mediate the gap effect? J ournal of Experimental Psychology: Human Perception and Performance, 19: 1251-1265.

10. Mayfrank $L$, Mobashery $M$, Kimming $H \&$ Fischer B (1986). The role of fixation and visual attention in the occurrence of express saccades in man. European Archives of Psychiatry and Neurological Sciences, 235: 269-275.

11. Reuter-Lorenz PA, Hughes HC \& Fendrich $R$ (1991). The reduction of saccade latency by prior offset of the fixation point: An analysis of the gap effect. Perception and Psychophysics, 49: 167-175.

12. Reuter-Lorenz PA, Oonk HM, Barnes LL \& Hughes HC (1995). Effects of warning signals and fixation point offsets on the latency of pro- versus antisaccades: implications for an interpretation of the gap effect. Experimental Brain Research, 103: 287-293.
13. Fendrich R, Hughes $H C \&$ Reuter-Lorenz PA (1991). Fixation-point offsets reduce the latency of saccades to acoustic targets. Perception and Psychophysics, 50: 383-387.

14. Kingstone A \& Klein RM (1993). What are human express saccades? Perception and Psychophysics, 54: 260-273.

15. Fischer B \& Rogal L (1986). Eye-handcoordination in man: a reaction time study. Biological Cybernetics, 55: 253-261.

16. Ross SM \& Ross LE (1981). Saccade latency and warning signals: effects of auditory and visual stimulus onset and offset. Perception and Psychophysics, 29: 429-437.

17. Rogal L, Reible G \& Fischer B (1985). Reaction times of the eye and the hand of the monkey in a visual reach task. Neuroscience Letters, 58: 127-132.

18. Fischer B (1989). Visually guided eye and hand movements in man. Brain, Behavior and Evolution, 33: 109-112.

19. Oldfield RC (1971). The assessment and analysis of handiness: The Edinburgh Inventory. Neuropsychologia, 9: 97-113. 